EchoGéo

ECHOGEO-Sur le Vif | 2012

\title{
La crise européenne. Un regard de géographe
}

\section{Yann Richard}

\section{OpenEdition}

Journals

\section{Édition électronique}

URL : http://journals.openedition.org/echogeo/13043

DOI : $10.4000 /$ echogeo. 13043

ISSN : 1963-1197

\section{Éditeur}

Pôle de recherche pour l'organisation et la diffusion de l'information géographique (CNRS UMR 8586)

\section{Référence électronique}

Yann Richard, «La crise européenne. Un regard de géographe », EchoGéo [En ligne], Sur le Vif, mis en ligne le 10 avril 2012, consulté le 10 décembre 2020. URL : http://journals.openedition.org/echogeo/ 13043 ; DOI : https://doi.org/10.4000/echogeo.13043

Ce document a été généré automatiquement le 10 décembre 2020.

\section{(c) ()) (9)}

EchoGéo est mis à disposition selon les termes de la licence Creative Commons Attribution - Pas d'Utilisation Commerciale - Pas de Modification 4.0 International 


\title{
La crise européenne. Un regard de géographe
}

\author{
Yann Richard
}

1 La crise et la description de ses effets économiques et sociaux occupent presque tout le champ médiatique ainsi qu'une bonne part de la littérature économique et politique (Chambon, 2009; Dervi, 2009; Sapir, 2008) depuis plusieurs années. L'élément déclencheur a été la crise des subprimes aux États-Unis, dont les conséquences ont été ressenties dans une grande partie du monde. Dans un deuxième temps, cette crise s'est muée en une crise grecque et européenne à cause de l'augmentation rapide des dettes souveraines. De nombreux intervenants sur les marchés financiers se demandent si les pays membres de la zone euro seront capables de rembourser une partie de leur dette. De fait, plusieurs pays européens sont très fragiles et empruntent sur les marchés à des conditions nettement moins avantageuses qu'autrefois.

2 La tempête que traverse l'Union européenne depuis 2008 n'est pas une simple dépression économique. Plus grave que celles qui l'ont précédée, elle a une signification profondément politique car elle menace la survie de l'Union elle-même. Cette dimension politique, voire géopolitique, a été étudiée abondamment par de nombreux auteurs, mais pas par les géographes sauf exception (Carroué, 2008, 2009a, 2009b ; Colin, 2010).

3 La crise met à mal l'intégration économique et monétaire que les Européens ont mis si longtemps à construire en exposant au grand jour les lignes de failles internes profondes qui la traversent. La Grèce ne doit pas être l'arbre qui cache la forêt. Le choc économique actuel ne fait que dévoiler des fragilités structurelles plus nombreuses et plus anciennes. Quelles sont-elles? Quel est leur impact sur l'intensité de la crise dans les différents pays ? Dans quelle mesure ces fragilités gênent-elles l'Union européenne et la zone euro pour sortir de la crise? L'Union n'est-elle pas parvenue au bout d'une logique qui s'essouffle en combinant de plus en plus difficilement intégration et éclatement?

On peut donner des éléments de réponse à ces questions en portant un regard de géographe sur cette dépression économique très grave et en prêtant attention à son déploiement dans l'espace européen. La principale faiblesse de l'Union européenne en cette période, ce sont ses divisions internes géopolitiques, à tel point qu'on peut se 
demander si l'appellation officielle «Union européenne » n'est pas un joli emballage qui cache une réalité moins attrayante. Elle s'est bel et bien construite comme un espace économique dont personne ne contexte l'existence, mais les dissensions nombreuses entre ses pays membres montrent qu'elle souffre entre autres d'une absence de leadership politique interne. Sortir de la crise suppose que des efforts de coordination soient faits. C'est d'autant plus difficile que, malgré plus de cinquante années d'intégration économique et politique, l'Union européenne se présente toujours comme une agrégation d'espaces nationaux, animés par des dynamiques pas toujours synchrones, et de pays dont les intérêts et les perceptions ne convergent guère dès lors qu'on regarde au-delà des grands principes.

\section{La crise européenne est-elle une crise de leadership?}

5 Pas une journée sans que l'Allemagne ne soit citée en modèle par une partie de la presse française et par certaines personnalités politiques. L'Allemagne est montrée comme l'exemple à suivre car ses performances macroéconomiques sont meilleures que celles des autres pays. Ces déclarations font en réalité écho à quelques très anciennes représentations: l'Allemagne exercerait une hégémonie économique sur l'Europe; elle aurait profité de l'effondrement du rideau de fer pour reconstituer son aire d'influence en Europe centrale et orientale; elle aurait imposé sa conception monétaire rigoriste au reste de l'Union européenne en influençant très clairement le pacte de stabilité qui accompagna la naissance de l'euro.

6 Aujourd'hui, on constate que l'Allemagne fait effectivement mieux que beaucoup de pays européens sur le plan économique. Sa balance des paiements courants est constamment excédentaire depuis $2002:+5,7 \%$ en 2010 , ce qui la place en $4^{\mathrm{e}}$ position dans l'UE (derrière le Luxembourg, les Pays-Bas et la Suède). Sa croissance économique annuelle, après avoir plongé en 2009, est de nouveau nettement supérieure à la moyenne de l'Union européenne : + 3,7 \% en 2010, contre $+2 \%$ pour l'UE et $+1,9 \%$ pour la zone euro. Enfin et surtout, elle semble beaucoup plus compétitive que les autres pays européen à cause de l'envolée de son solde du commerce extérieur. Par ailleurs l'Allemagne est un des quelques pays européens qui ont conservé une AAA pour leur dette souveraine auprès des agences de notation. 


\section{Illustration 1 - Balance des paiements courants}

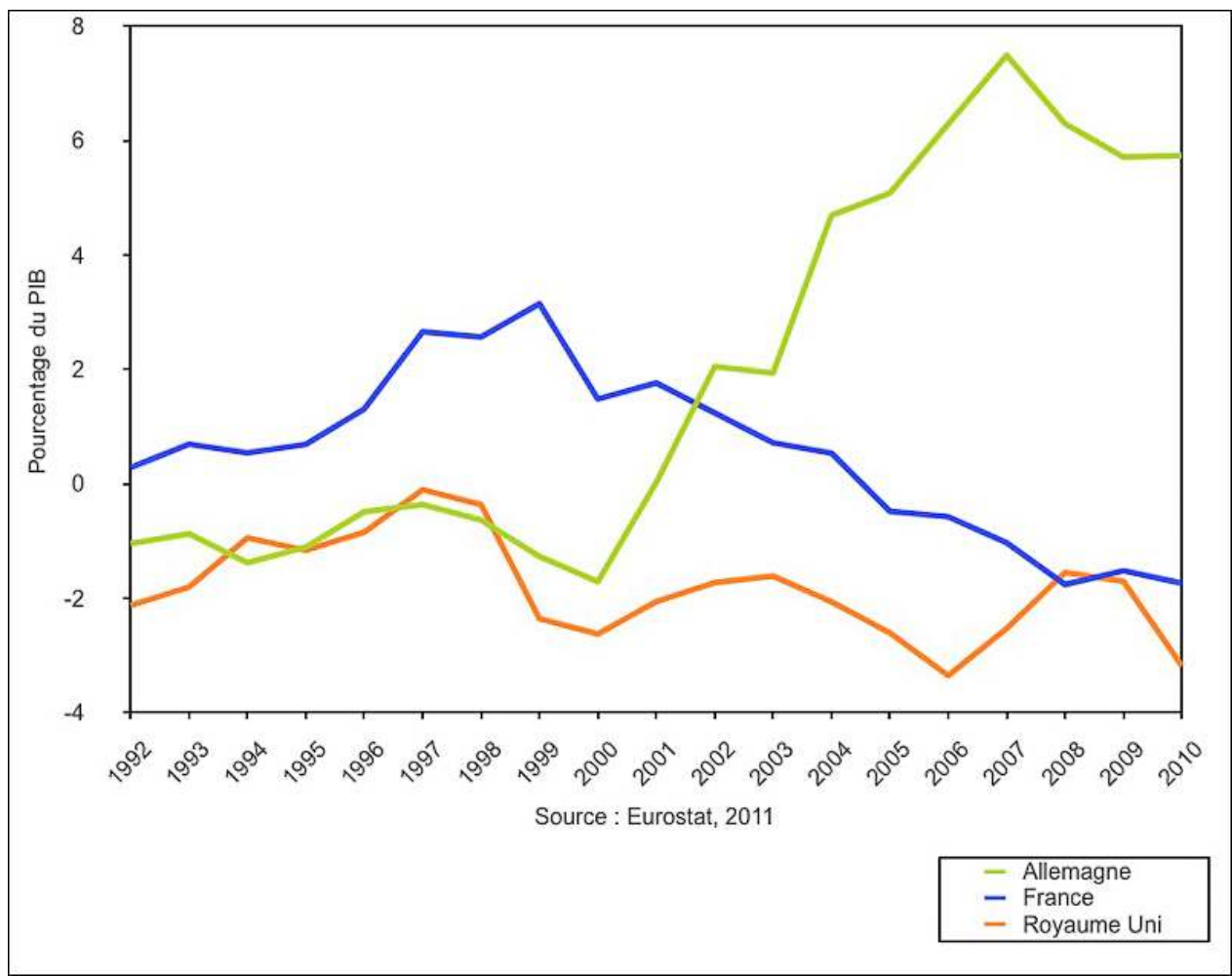

Source : Eurostat, 2011.

Illustration 2 - Balance du commerce extérieur des biens et services

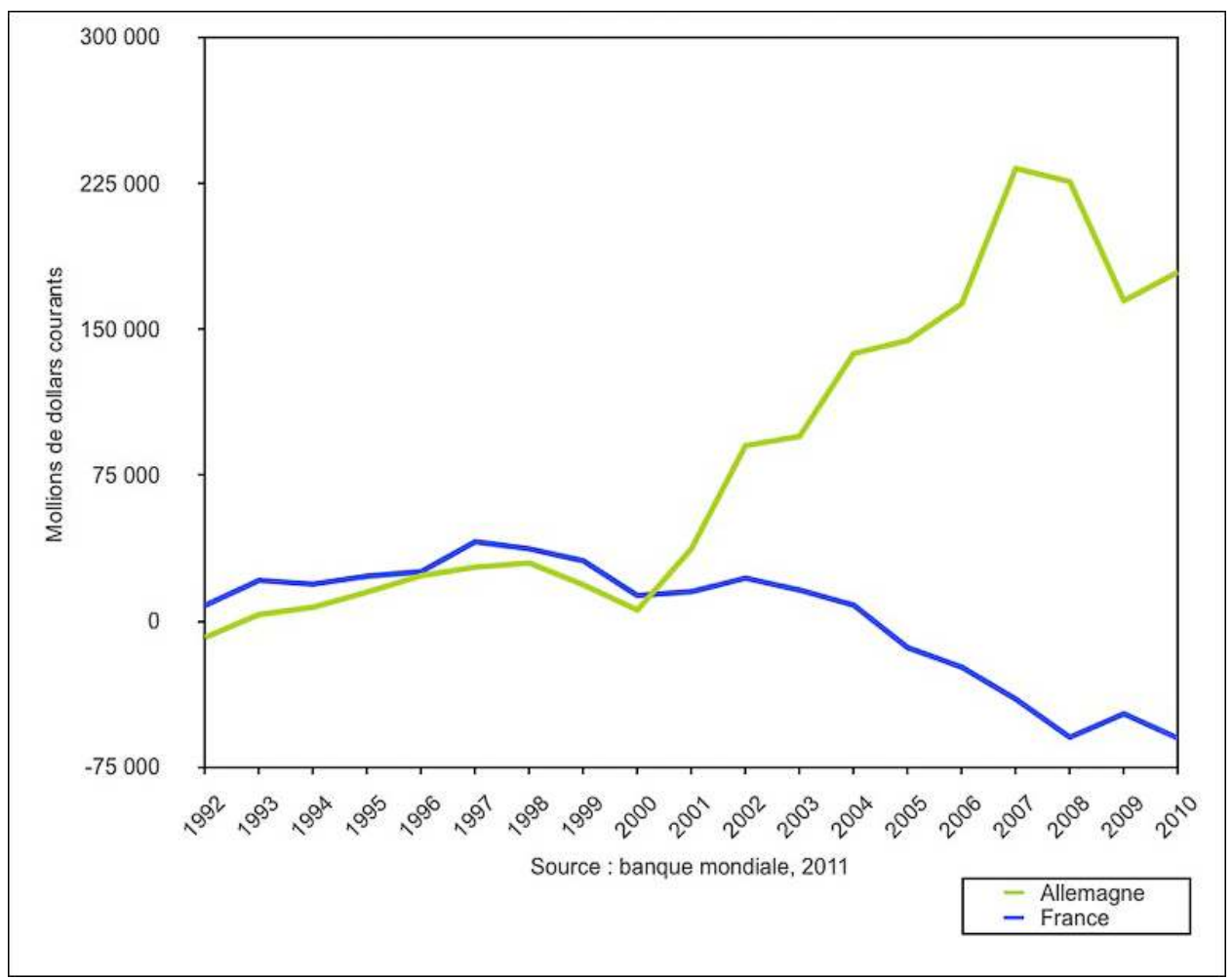

Source : Banque mondiale, 2011 
Ces performances sont source d'inquiétude en France dont on se demande si elle est encore en mesure de jouer son rôle traditionnel dans le couple leader européen. Il faut dire que les chiffres sont moins flatteurs que ceux de l'Allemagne, qui pèse à elle seule $21 \%$ du revenu national brut de l'Union européenne, contre $17 \%$ pour la France. Un déséquilibre macroéconomique accru entre les deux pays se traduirait par un affaiblissement politique, suscitant la crainte de voir se germaniser l'Europe.

Plusieurs éléments poussent à nuancer voire à remettre en question l'idée d'un risque d'hégémonie allemande. Premièrement, les performances macroéconomiques de l'Allemagne n'ont pas été toujours aussi bonnes. Ce sont des décisions politiques douloureuses prises par le gouvernement Schröder qui ont permis de renverser une tendance autrefois mal orientée : la balance des paiements a été négative jusqu'en 2000. De plus, la croissance allemande est fragile : largement supérieure à celle de la France en 2010, elle est en réalité un peu plus faible sur l'ensemble de la période 2002-2010 en moyenne annuelle ( $0,9 \%$ pour l'Allemagne et $1,1 \%$ pour la France) et nettement inférieure à celle de l'UE (1,3\%). Par ailleurs, la productivité de sa main-d'œuvre est inférieure. La France est en $4^{\mathrm{e}}$ position dans l'UE (et $6^{\mathrm{e}}$ mondiale), alors que l'Allemagne vient seulement à la $13^{\mathrm{e}}$ place avec un fort écart: la quantité de PIB produite par personne active en France dépassait 55000 dollars en 2008, contre 42500 pour l'Allemagne, ce qui place cette dernière en dessous de la moyenne de la zone euro. Enfin et surtout, le poids relatif de l'Allemagne dans le revenu national brut de l'UE est en baisse relative depuis les années 1990 (23 \% en 1992, 21 \% en 2010).

Deuxièmement, l'Allemagne a fait des choix politiques qui se sont révélés payants sur le court terme mais qui pourraient ne pas être soutenables sur le long terme et mettre ses partenaires européens en situation délicate. Dans les années 2000, le chancelier G. Schröder a mis en œuvre une politique de rigueur salariale afin de relancer la compétitivité allemande, c'est-à-dire sa capacité à prendre des parts de marché à l'extérieur. La balance commerciale allemande a ainsi atteint des excédents sans précédent à la fin des années 2000. Mais, dans le même temps, la balance commerciale française se dégradait et semble désormais structurellement déficitaire, notamment à cause d'un déficit croissant avec l'Allemagne. Pourtant, et c'est là le paradoxe, les performances économiques globales mesurées par la croissance du PIB ont été légèrement supérieures en France sur la même période. En fait, le paradoxe s'explique aisément : ce que l'Allemagne gagne sur les marchés extérieurs, elle le perd à l'intérieur où la demande est minée par des salaires stagnants. Il est difficile de savoir si le bilan de ces deux effets contradictoires est positif ou négatif pour l'Allemagne. Quoi qu'il en soit, un pays peut difficilement jouer un rôle de leader s'il n'est pas en mesure d'être une locomotive économique pour l'ensemble de ses partenaires. Par ailleurs, si les autres États européens menaient des politiques aussi rigoureuses, c'est toute la demande européenne qui serait déprimée. Cela pèserait sur la croissance économique européenne globale, dans la mesure où l'Europe reste un ensemble économique relativement fermé vis-à-vis du reste du monde et dépend donc d'abord de sa demande intérieure. L'austérité salariale est en partie responsable des déséquilibres de l'Union européenne.

Troisièmement, l'Allemagne n'est pas monolithique sur le plan politique. La chancelière Merkel a fait jusqu'à présent le choix de l'austérité, en continuité avec l'administration précédente, en exportant ce principe politique dans les pays européens en difficulté, soutenue il est vrai par une bonne partie de son opinion publique. Mais certains partis, actuellement dans l'opposition, ne sont pas d'accord avec son traitement politique de la 
crise. S'ils arrivaient au pouvoir, les Verts et les sociaux-démocrates pourraient œuvrer en faveur de la mutualisation des dettes souveraines et permettre de nouveaux transferts de souveraineté vers les institutions européennes. Quoi qu'il en soit, la majorité gouvernementale allemande actuelle se cabre sur le principe de stabilité financière, ce qui a pour effet de susciter au mieux des craintes, au pire un sentiment de rejet dans d'autres pays européens, notamment en Grèce.

On se trouve bien dans une situation où l'Union européenne souffre d'un manque de leadership. Par définition, un pays hégémonique est un fournisseur - généralement gratuit - de stabilité et de sécurité (Battistella et alii, 2006). Il peut éventuellement agir comme prêteur en dernier ressort (Kebabdjian, 1999), ce qui lui confère son statut de garant de l'ordre établi. A l'heure actuelle, l'Allemagne n'est pas en mesure d'exercer ce leaderhip car ses propositions n'entraînent pas l'adhésion de ses partenaires. Et sa situation est d'autant plus difficile que son partenaire habituel, la France, est affaibli économiquement donc politiquement. L'Allemagne est d'ailleurs plus un membre hésitant qu'un leader. Elle a infléchi sa position sur l'idée de gouvernance économique dont elle ne voulait pas entendre parler initialement. Mais il est difficile de fonder sur le long terme un statut de leader sur le seul principe de la discipline budgétaire et l'austérité, pour plusieurs raisons : les effets de ces choix politique sur la croissance seront sans doute négatifs; beaucoup d'autres pays européens ne font pas la même analyse; la croissance rapide des dettes souveraines de plusieurs pays est au moins autant une conséquence qu'une cause de la crise actuelle.

\section{L'Union européenne comme collection de territoires nationaux et d'économies nationales}

12 La désunion européenne face à la dépression nous rappelle que l'UE est encore une collection de réalités nationales, d'espaces aux dynamiques sociales et économiques propres. Même si l'intégration a fait de grands progrès depuis les années 1980 (Acte unique, Maastricht, traité constitutionnel, etc.), elle reste divisée. Des lignes de discontinuité traversent l'Union européenne. Certaines sont anciennes et structurelles et soulignent avec force la persistance de la désunion politique de l'Europe. Ces discontinuités sont plus révélées que produites par la crise actuelle. La gravité de la situation économique y trouve peut-être même une partie de ses causes.

\section{La dépression économique pour tout le monde?}

Les différences régionales de performance économique sont soulignées quotidiennement avec force dans la presse. Les pays du nord et du nord-ouest de l'Europe se distinguent par leurs résultats flatteurs dans de nombreux domaines. Leurs taux de chômage sont généralement inférieurs à la moyenne de l'Union européenne (8,9\% en 2010) : 8,2\% pour la Finlande et la Suède, 7,9 \% pour la Belgique, $6 \%$ pour le Danemark, 3,4 \% pour les PaysBas. Leur productivité est généralement élevée. Et leur balance des paiements courants est structurellement excédentaire sur le temps long. Toutefois, leurs performances en terme de croissance sont inégales : la Suède, la Finlande, le Luxembourg et la Belgique ont eu une croissance supérieure à la moyenne européenne, mais le Danemark et les Pays-Bas sont en dessous; par ailleurs, ils font nettement moins bien que les PECO sur l'ensemble de la période 2002-2010. 
Illustration 3 - Balance des paiements courants des pays méditerranéens de l'Union européenne

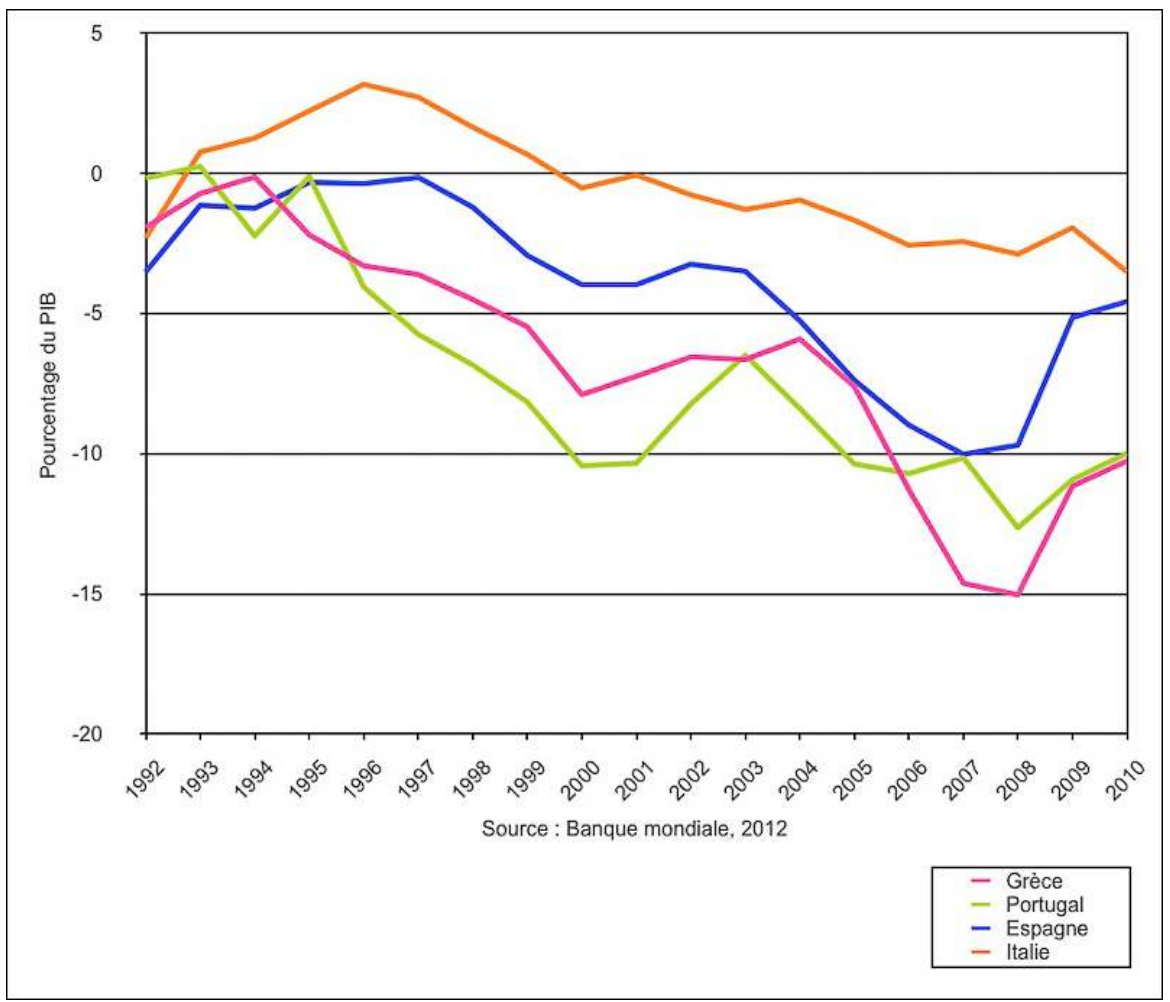

Source : Banque mondiale, 2012

14 A l'inverse, les pays du sud de l'Europe présentent des indicateurs macro-économiques souvent moins bons. L'Espagne ( $16^{\mathrm{e}}$ position dans l'Union européenne), la Grèce $\left(17^{\mathrm{e}}\right)$, Chypre $\left(19^{e}\right)$, Malte $\left(20^{e}\right)$ et le Portugal $\left(22^{e}\right)$ ont une productivité du travail inférieure à la moyenne européenne, mais l'Italie est juste au-dessus. La balance des paiements courants est déficitaire dans tous les pays méditerranéens, qui sont les derniers du classement dans l'UE avec la Roumanie et la Pologne. A l'exception de l'Italie, ces pays ont une balance constamment déficitaire depuis une vingtaine d'année. La crise n'est pas toujours responsable d'une situation que l'on peut qualifier de structurelle. Elle a simplement accentué les difficultés de pays déjà fragiles dans les années 1990. Enfin, l'Espagne a un taux de chômage structurellement plus élevé que la moyenne de l'UE depuis les années 1990 : plus de $15 \%$ entre 1990 et 2010 pour une moyenne européenne de $9,2 \%$. Toutefois, dans ce dernier cas, la crise économique a une part de responsabilité car le chômage avait reculé sensiblement à partir du milieu des années 1990, pour exploser à nouveau en 2009 : $22,4 \%$ en $1993,8,3 \%$ en $2007,18,3 \%$ en 2009.

Les nouveaux pays membres de l'Europe centrale et orientale sont également dans une situation délicate. Sur le temps long et dans la crise, ils ne forment pas un ensemble homogène car leurs performances économiques sont contrastées. Leur productivité est en général faible : dans les $10 \mathrm{PECO}$, elle connait une croissance régulière depuis le début des années 1990, mais elle reste inférieure à la moyenne de l'UE. Seule l'Estonie s'en rapproche. La Roumanie, la Bulgarie, la Hongrie, la Pologne, la République tchèque, la Lituanie et la Lettonie occupent les dernières places du classement européen. Pour les autres indicateurs, les PECO sont dispersés. Certains ont des taux de chômage très supérieurs structurellement à la moyenne européenne (Slovaquie, Pologne, Bulgarie, 
Lituanie, Lettonie), d'autres sont en dessous (Estonie, Hongrie, Roumanie, République tchèque). D'une façon générale, ils sont parmi les pays européens qui souffrent le plus de la dépression économique avec un effondrement inquiétant de leur PIB en 2009 (- $18 \%$ en Lettonie, un des records du monde, $-13,9 \%$ en Estonie et - $14,7 \%$ en Lituanie $\left.^{1}\right)$, à l'exception de la Pologne dont l'évolution du PIB est toujours restée positive.

Ces évolutions aussi inquiétantes que brutales ont été en partie causées par la dépendance de ces économies vis-à-vis des capitaux étrangers, venus pour l'essentiel des banques de l'Europe occidentale. Les effets de la crise sont spectaculaires sur les entreprises et sur le niveau de vie des habitants de ces pays, surtout dans les pays baltes, qui ont souvent contracté des emprunts libellés en euros et qui ont dû faire face à un renchérissement souvent insupportable du crédit. Certains se distinguent enfin par la vitesse de leur récupération : la Slovaquie, l'Estonie et la République tchèque sont passées de taux négatifs à des taux positifs entre 2009 et 2010. Le cas le plus spectaculaire est celui de l'Estonie qui est passée de $-13,9 \%$ à $+3,1 \%$ en un an. Ce pays, dont les finances publiques étaient en bon état avant la crise, a mis en œuvre une politique d'austérité d'une extrême dureté, comme la Lettonie, et a réussi à entrer dans la zone euro au plus fort de la crise. Au total, les nouveaux pays membres de l'UE (hors Chypre et Malte) peuvent donc être répartis dans plusieurs groupes: les pays baltes ont souffert de leur grande dépendance vis-à-vis des capitaux étrangers; les pays de l'Europe centrale ont moins durement ressenti la crise, à l'exception de la Hongrie et de la Roumanie, mais ils ont dû faire face à une contraction sérieuse de leurs exportations ; l'économie polonaise se distingue car elle a continué de croître grâce à la vivacité de son marché intérieur.

Dans ce tour d'horizon européen, on peut se demander si la géographie de l'euro est corrélée à celle de la dépression économique. En d'autre termes, vaut-il mieux faire partie du club ou vaut-il mieux ne pas en être pour passer à travers les difficultés ? L'euro est-il une protection ou un facteur aggravant? La question mérite d'être posée car plusieurs partis politiques ont proposé en Europe de sortir de l'euro. La réponse n'est pas aisée car la géographie de l'intensité de la crise et de la vigueur de la reprise ne sont pas corrélées à celle de la zone euro. De nombreux pays non membres de la zone euro ont des performances économiques peu enviables : le Royaume-Uni a une balance des paiements constamment déficitaire depuis longtemps, de même que la République tchèque, la Pologne, la Roumanie et la Bulgarie. Inversement, certains pays de la zone euro sont excédentaires : Finlande, Autriche, Allemagne, Pays-Bas et Luxembourg. On peut en dire autant à propos du chômage : certains pays membres de la zone euros sont en mauvaise posture (Espagne, Slovaquie, France, Finlande, Italie), alors que d'autres ont peu de chômeurs (Danemark, Pays-Bas, Autriche, Luxembourg). Inversement, les pays non membres se retrouvent aussi bien parmi les pays à fort taux de chômage que dans ceux où le chômage est peu élevé. Enfin, le tableau est aussi mêlé en ce qui concerne la croissance, dont le rythme n'est pas clairement corrélé à la possession de l'euro. Entre 2002 et 2010, parmi les pays à forte croissance, on trouve autant de membres que de non membres de la zone euro. L'actualité récente va dans le même sens puisque le Royaume-Uni, non membre, a mis en œuvre une politique d'austérité presque aussi rigoureuse qu'en Grèce. Le Royaume-Uni ne semble pas subir les effets de l'augmentation spectaculaire de sa dette publique, mais certains pays de la zone euro non plus : malgré leur récente dégradation, la France, l'Italie et l'Espagne ont réussi à placer récemment de la dette à des taux avantageux et parfois inférieurs à leurs niveaux antérieurs. Enfin, la géographie de l'évolution du revenu national brut par habitant n'est pas non superposable à celle de 
l'euro: il a fortement chuté au Danemark et au Royaume-Uni par exemple et il a augmenté ou s'est maintenu en Autriche, aux Pays-Bas, en Slovaquie et en Allemagne. Tout porte à penser que les évolutions économiques des pays membres de l'UE sont essentiellement nationales.

En bref, la géographie de l'intensité de la crise n'est pas surprenante car elle est assez bien corrélée avec celle de la solidité structurelle des économies de l'Union européenne.

Sur la carte présentée en illustration 4, on a représenté les pays membres de l'UE en fonction de leurs performances macroéconomiques dans six domaines par rapport à la moyenne de l'Union européenne: taux de chômage, balance des paiements courants, solde commercial, croissance annuelle, productivité de la main-d'œuvre, dette. Il s'agit des performances annuelles moyennes sur la période 2002-2008, c'est-à-dire avant que les effets de la dépression économique soient observables en Europe. Pour chaque indicateur, on a classé les pays de la façon suivante: positivement lorsque leur performance moyenne était supérieure à la performance moyenne de l'UE 27 ; négativement lorsqu'elle était inférieure.

Illustration 4 - Performances macroéconomiques des pays membres de l'UE 27 actuelle

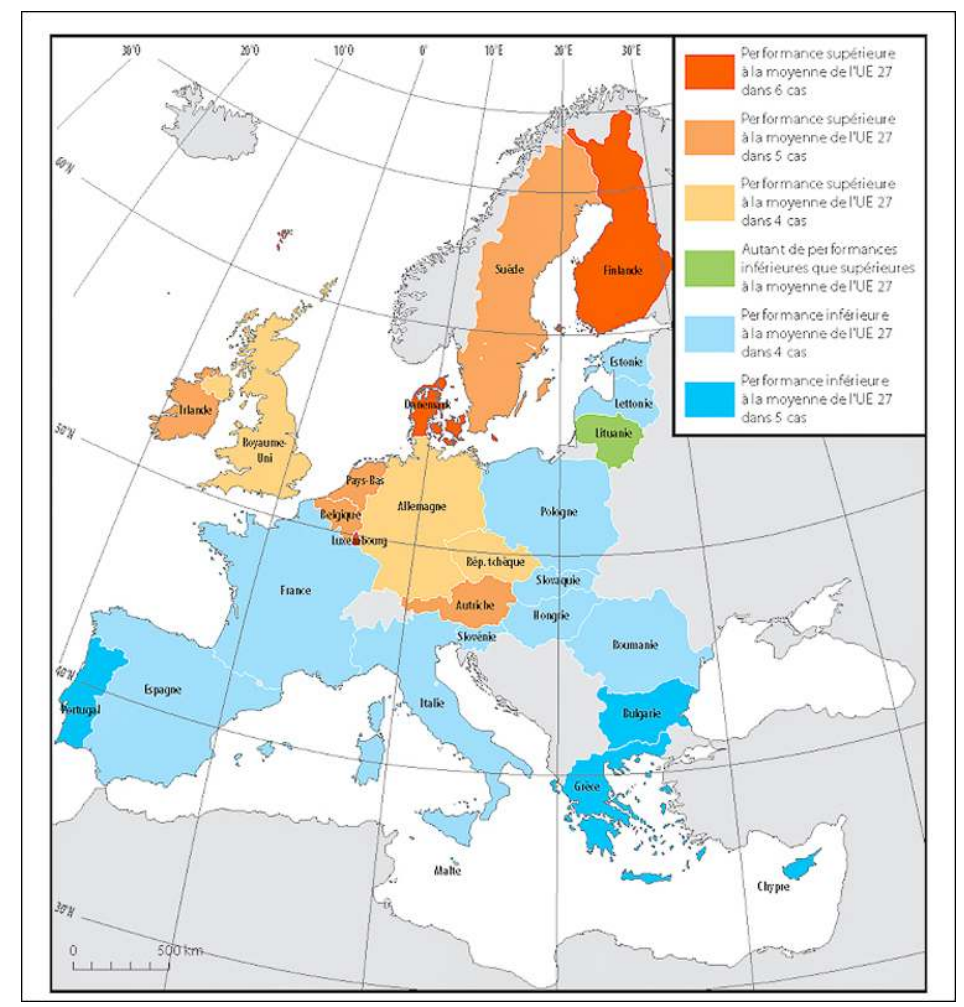

Source : Eurostats 2012 ; conception : Yann Richard, réalisation : Florence Bonnaud.

La carte est sans appel. Les économies structurellement les plus solides sont principalement celles du nord, du nord-ouest de l'Union européenne: Danemark, Finlande, Luxembourg, Belgique, Pays-Bas, Suède, Allemagne. A l'inverse, ce sont surtout des économies du sud et des pays de l'Europe centrale et orientale qu'on retrouve dans l'Europe fragile, en particulier la Bulgarie, Chypre, la Grèce et le Portugal. 


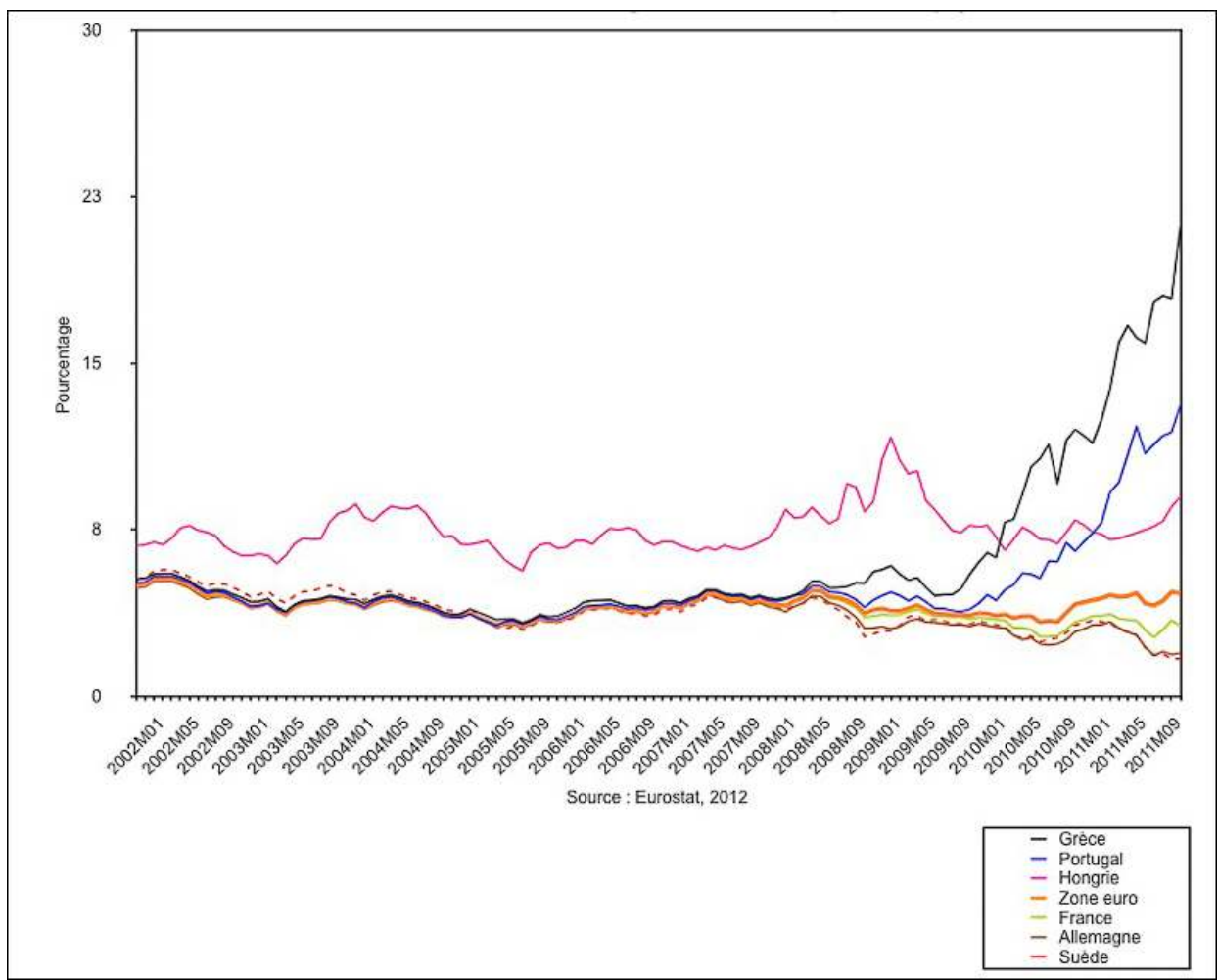

Source : Eurostat, 2012.

21 Les inégalités nationales de performance économique sont mises en exergue par l'évolution des taux de rendements des obligations d'État à 10 ans sur les marchés financiers. Le graphique ci-dessous montre plusieurs faits importants :

- les taux d'intérêt à 10 ans sur les obligations d'État étaient compris entre 4,84 \% et 8,26\% en janvier 2002 et ils sont restés très proches jusqu'en mai 2008. C'est à l'été 2008 qu'on observe une divergence soudaine des conditions d'emprunt;

- la Hongrie est perçue comme une économie fragile depuis longtemps et doit supporter des taux d'intérêt plus élevés que la moyenne de la zone euro ;

- les taux appliqués aux emprunts grecs ont connu un premier décrochage en septembre 2008. C'est dire que les acteurs financiers ont pointé du doigt non pas la fragilité de ce pays, connue depuis très longtemps, mais la fragilité de la zone euro dans son ensemble: en appliquant des conditions d'emprunt plus dures à la Grèce, ils ont simplement montré leurs doutes sur la capacité des autres pays de la zone à servir de garde-fou car certains se sont révélés eux aussi fragiles ;

- la situation portugaise est très préoccupante également puisque au Portugal les taux d'emprunt ne cessent de croître depuis l'hiver 2009-2010 ;

- d'autres États voient leurs taux d'emprunt augmenter et décrocher par rapport à la moyenne européenne depuis la crise : la Slovénie, l'Irlande, l'Italie, la Lettonie, etc. ;

- inversement, les pays du Nord de l'Europe, voient leurs taux décrocher vers le bas : la Suède, le Danemark, l'Allemagne, le Royaume-Uni (pour combien de temps ?), le Luxembourg, les Pays-Bas et la Finlande, confirmant la solidité perçue et sans doute réelle de ces économies.

Il y a une forte corrélation entre la géographie des taux à long terme et la carte des forces et faiblesses des économies européennes avant le sommet de la crise. En d'autres termes, 
les marchés financiers ne font que s'engouffrer là où des faiblesses existent depuis longtemps.

\section{La persistance des dynamiques nationales}

23 Cette conclusion ne surprendra pas ceux qui s'intéressent à l'Union européenne depuis longtemps. Malgré d'indéniables avancées dans le processus d'intégration, en particulier depuis l'Acte unique et la ratification du traité de Maastricht, il est clair que les dynamiques spatiales qui animent les pays membres sont principalement nationales. En d'autres termes, il existe encore de forts effets frontières dans l'Union.

Avec l'intégration européenne, la montée en puissance des réseaux métropolitains, les diverses formes de la libéralisation et de la dérégulation économiques et enfin les mouvements de décentralisation, on aurait pu s'attendre à voir les États perdre de leur importance en tant qu'acteur de la régulation et de la répartition des activités économiques. Mais qu'en est-il réellement? Les nations perdent-elles leur importance par rapport à d'autres territoires, devenus plus pertinents? En réalité, les dynamiques économiques régionales et urbaines semblent avoir été largement déterminées par les nations dans lesquelles ces villes et ces régions sont situées. Selon une étude menée sur les villes européennes, $74 \%$ des écarts de croissance économique entre les villes sur la période 1995-2006 étaient liés aux pays auxquels ces villes appartenaient (Van Hamme, 2011). Autrement dit, si les villes allemandes ou italiennes ont eu des dynamiques économiques plutôt médiocres pendant cette période, elles l'ont dû à la faiblesse de la croissance économique nationale italienne et allemande. A l'inverse, les dynamiques fortes de l'économie espagnole se sont retrouvées dans l'ensemble des villes espagnoles. A l'ère des réseaux interurbains et de la mondialisation, les différences entre les nations demeurent déterminantes, y compris dans un marché fortement intégré comme le marché européen.

25 La crise financière et économique de 2008-2009 a remis en lumière le rôle décisif des États, apparemment seuls capables de remettre en marche un système qui partait à la dérive (Sapir, 2008). En fait, les États conservent les principaux instruments de la régulation économique en Europe, que ce soit la régulation du marché du travail, les politiques fiscales, les mécanismes de redistribution sociale, etc. Seules les politiques monétaires leur ont en partie échappé - mais uniquement pour les pays de la zone euro sans toutefois qu'une véritable politique économique accompagne les décisions prises par la Banque centrale européenne (BCE) dans la gestion de l'Euro. Pour résumer, bien qu'affaiblis par le décalage entre leur capacité d'action au niveau national et l'action internationale des grands acteurs économiques privés, les États européens restent encore les acteurs clés de la régulation économique (Boyer, 2003).

\section{L'écart est-ouest : une très vieille histoire}

Malgré sa prospérité d'ensemble, l'Europe est traversée par des inégalités territoriales. La division est/ouest est de loin la plus profonde. Elle se reflète à travers de multiples indicateurs économiques, sociaux et démographiques. Dès le XVI siècle s'est mise en place une division du travail au sein de l'espace européen. Les pays d'occident connaissent un processus d'industrialisation et participent plus ou moins directement à l'expansion de l'Europe en dehors de ses frontières, tandis que l'Europe de l'est se 
spécialise dans la production de céréales et d'autres matières premières à travers la mise en place de grandes propriétés seigneuriales et du "second servage». Ce clivage est/ ouest s'est figé après la guerre de part et d'autre du « rideau de fer ».

La différence est/ouest est donc bien une division profonde et ancienne de l'espace européen, plusieurs fois réactualisée par des ruptures majeures. D'une part, le rideau de fer prolonge des inégalités de très long terme. D'autre part, il met en place une ligne de séparation très profonde là où existaient de larges espaces de transition entre la prospère Europe occidentale et l'Europe orientale. On constate ainsi que cet espace de transition entre l'est et l'ouest de l'Europe semble s'être reconstitué rapidement après la chute du communisme. Malgré les réformes post socialistes, on retrouve en 2008 des écarts de niveaux de PIB par habitant assez proches de ceux qui existaient au début de l'ère communiste, les pays proches de l'Europe occidentale (Tchéquie, Slovénie, Hongrie) atteignant des niveaux bien supérieurs aux pays situés plus à l'est (Roumanie, Bulgarie). Pourtant, au cours de la période communiste, les niveaux de PIB par habitant avaient fortement convergé, les pays les plus pauvres ayant connu les plus fortes croissances jusque dans les années 1970. Tout se passe comme si, après la chute des régimes communistes, tout était rentré dans l'ordre et que les écarts initiaux avaient été rétablis, reconstruisant cet espace de transition entre l'ouest et l'est de l'Europe.

D'ailleurs, les croissances économiques de la décennie 2000 doivent être relativisées. Dans de nombreux cas, elles ne sont que le rattrapage du niveau de richesse que ces pays avaient atteint à la fin de l'époque communiste. De plus, beaucoup de ces pays ont été très touchés par la crise de 2008 - 2010, les ramenant plusieurs années en arrière en ce qui concerne leur niveau de PIB par habitant. Mais, plus fondamentalement encore, ces pays gardent des faiblesses structurelles importantes: ils ont peu de mainmise sur leurs activités économiques car celles-ci sont largement contrôlées par des sociétés occidentales; leur croissance s'appuie uniquement sur le développement de secteurs à faibles ou moyennes technologies (selon les pays), ce qui les rend très vulnérables à des processus de délocalisation dont ils ont, il est vrai, largement bénéficié au cours des années 2000. Il y a certes eu des remontées technologiques dans les types d'investissements réalisés, mais il existe encore un fossé avec les économies d'Europe occidentale où se concentrent la capacité de contrôle et la maîtrise technologique.

\section{Une traditionnelle disparité centre - périphérie}

L'Europe est traversée par une opposition structurelle forte et ancienne entre le centre et la périphérie (Van Hamme, 2011). Le centre se caractérise par de fortes densités d'hommes et d'activités, une grande prospérité, la concentration des activités de commandement et la maîtrise technologique. Là se concentre l'essentiel de l'activité économique, dans une dorsale qui va du nord-ouest de l'Angleterre au centre de l'Italie en passant par le Benelux, l'Allemagne, le nord et l'est de la France et la Suisse. Dans cet espace, les villes grandes, moyennes et petites sont nombreuses, séparées par de faibles distances; l'agriculture est intensive depuis des siècles, ce qui permet d'atteindre des densités de population plus élevées que dans le reste de l'Europe ; la finance et les sièges sociaux des grandes multinationales sont concentrés dans quelques métropoles (Francfort, Paris, Londres, Amsterdam etc.) ; l'industrie de moyenne et haute technologie est surreprésentée, etc. À l'inverse, les périphéries européennes (Irlande, péninsule ibérique, sud de l'Italie, Balkans) se caractérisent par des densités de populations et 
d'activités plus basses, une agriculture moins intensive, une spécialisation dans des activités agricoles et industrielles à plus faible valeur ajoutée et la rareté des fonctions stratégiques, comme les sièges sociaux des grandes entreprises.

Cette opposition structurelle est ancienne. Dès le Moyen-âge, les historiens soulignent l'existence de zones plus denses et économiquement plus actives au cœur de l'Europe, en particulier la Flandre historique et l'Italie du nord et du centre (Braudel, 1979). Dès la fin $\mathrm{du} \mathrm{XV}^{\mathrm{e}}$ siècle, les indices de l'existence d'une dorsale européenne plus innovante sont déjà présents. La géographie des imprimeries à cette époque montre en creux de façon spectaculaire le caractère sous-développé de l'Europe de l'est à l'exception de l'actuelle Tchéquie. Ces disparités furent creusées davantage pendant la Révolution industrielle qui fut le point de départ d'une rapide concentration spatiale de la production et de la population. On assista à une concentration de l'industrie dans les pays du nord-ouest de l'Europe qui connaissaient une révolution industrielle précoce et, au sein de ceux-ci, dans les grandes régions industrielles et les grandes villes riches en activités de service.

31 En 2010, les écarts de développement et plus encore de structures restent importants entre le centre et les périphéries de l'Europe. À l'exception des grandes villes, les régions périphériques ont encore des niveaux de PIB par habitant inférieurs de 30 à $50 \%$ à ceux de nombreuses régions centrales. Surtout, leurs structures économiques restent plus faibles, avec la spécialisation de l'activité dans les services banalisés (commerce, hôtelsrestaurants-cafés, services non marchands etc.) ou dans l'industrie à faible valeur ajoutée. Plus encore, au cours des années 2000, les performances économiques élevées de pays comme la Grèce, l'Irlande ou l'Espagne - voire de certains pays de l'est comme les pays baltes - se sont appuyées sur une forte consommation intérieure, en particulier dans le domaine immobilier, avec un rapide endettement des ménages et/ou de l'État. En revanche, il y a peu de signes, sauf en Irlande, de véritables remontées des filières technologiques au cours des années 2000 , c'est-à-dire d'un passage progressif vers des technologies de plus en plus complexes dans les processus de production. Tout se passe comme si ces économies se trouvaient dans une situation d'entre-deux, incapables de faire face à la concurrence des pays de l'est dans les technologies moyennes ou de l'Asie dans les industries à forte intensité de main-d'œuvre, mais incapables aussi de se situer au niveau des pays d'Europe du nord en matière de maitrise technologique.

\section{Quels scénarios de sortie de crise ? Eclatement de I'Union, statu quo ou intégration renforcée}

Les disparités internes de l'UE associées à la profonde crise actuelle amènent à se poser très sérieusement la question de l'avenir de la communauté. Trois scénarios existent: l'éclatement de l'union monétaire et peut-être de l'Union européenne elle-même; le rafistolage de l'Union au nom d'un maintien précaire de ce qui est acquis ; la relance du processus d'intégration selon différentes formes. Le troisième scénario est le plus attrayant, mais même ses plus fervents partisans doivent admettre que de nombreux obstacles existent. 


\section{Crise et cacophonie à l'échelon gouvernemental}

33 Il faudrait être aveugle pour ne pas se rendre compte que les pays européens ont à ce jour répondu dans le désordre à la crise économique et à la crise de la dette. Parmi beaucoup d'autres, deux facteurs internes à l'Union européenne contribuent à la fébrilité des marchés financiers et à la fragilité de l'euro: la divergence des positions des États à propos des solutions à la crise et, par conséquent, la lenteur des décisions et de leur mise en œuvre. Si le cas irlandais a été traité relativement vite, il a fallu un long laps de temps pour que les pays membres de l'UE se mettent en ordre de bataille après la prise de conscience de l'ampleur des problèmes de la Grèce. C'est seulement le 21 février 2012 qu'un plan de secours crédible a été adopté. Mais avant cela, il a fallu franchir de nombreux obstacles et de nombreuses oppositions internes pour créer le fonds de secours européen, remplacé à terme par le mécanisme européen de solidarité financière. Bien que les Européens aient pris ces décisions ensemble, les marchés ne sont pas rassurés car il a fallu forcer la main de pays membres plus riches qui ne voyaient pas pourquoi ils devaient payer pour les plus pauvres et les moins vertueux.

L'Union européenne, principalement la zone euro, semble avancer dans le sens d'une plus grande intégration en renforçant son union budgétaire ${ }^{2}$. Le pacte de discipline budgétaire décidé par les ministres européens en janvier 2012 instaure quelques nouveautés: il donne un caractère officiel aux sommets de la zone euro (qui devront se tenir au moins deux fois par an), pour garantir aux investisseurs que la monnaie européenne sera soutenue collectivement et que les politiques économiques des pays membres seront plus coordonnées; les États devront signaler à l'avance leurs émissions obligataires ainsi que tout projet de réforme économique majeure ; ils s'engagent à coordonner leurs actions si nécessaire. Pour mettre en oeuvre ces politiques, les États de la zone euro se réuniront " au moins deux fois par an »; le Parlement européen sera associé aux travaux de la zone euro par le biais d'une conférence réunissant les présidents des commissions budgétaires des États et du Parlement européen; la Cour européenne pourra sanctionner financièrement les États qui auront un déficit supérieur à $3 \%$ du PIB, etc.

Toutefois, ces mesures sont fragilisées par l'existence de profondes divisions. Des positions divergentes se sont exprimées par exemple à propos de la taxe sur les opérations financières : le Danemark est favorable à l'idée d'imposer le secteur financier ; le Luxembourg se méfie; les Pays-Bas sont radicalement contre; les Britanniques demandent l'arrêt de toutes les discussion sur une mesure qu'ils présentent comme inutile et inefficace; les Allemands et les Français sont pour... Ces divergences sont si fortes que la taxe n'existera pas avant longtemps. Les divisions ne sont pas moins évidentes à propos du pacte européen de discipline budgétaire : à terme, on ne tolérera plus qu'un «déficit structurel »-hors effet de la conjoncture économique - inférieur à $0,5 \%$ du PIB. Or la notion de déficit structurel fait débat dans l'Union, tant sur sa définition que sur sa pertinence. La crédibilité de la mesure est donc faible avant même son entrée en application. De plus, certaines oppositions existent. Le Royaume Uni a refusé d'adhérer à cette règle. Il est vrai qu'il n'est pas membre de la zone euro mais cela renforce l'impression de division de l'Union. La République tchèque a refusé de s'engager dans cette voie, mettant en avant des obstacles institutionnels. La Pologne, craignant sans doute de voir se mettre en place une sorte de club exclusif de l'euro, a exprimé son désir de participer aux sommets des pays de la zone. Enfin, l'idée allemande d'instaurer une 
tutelle budgétaire sur la Grèce n'a pas été soutenue par les 24 autres pays signataires du pacte. Mais il est vrai que cette tutelle existe déjà de facto.

On voit bien que depuis le début de la crise, les pays membres de l'UE sont rétifs à l'idée de fédéralisation. Cela se traduit par la méthode qu'ils ont adoptée pour répondre aux soubresauts de la conjoncture. Le traitement de la crise se fait de façon intergouvernementale, c'est-à-dire à l'unanimité. De cette façon, les pays membres espèrent ne pas s'engager trop loin dans un domaine qui touche à leurs compétences régaliennes. Plusieurs questions se posent alors. Combien de temps l'UE, et a fortiori la zone euro, pourront continuer à fonctionner politiquement sur un mode intergouvernemental alors que l'intégration est de facto très avancée ? Combien de temps pourront-ils continuer à mener des politiques non coordonnées dans des domaines cruciaux tels que la fiscalité et le droit du travail? En fait, le choix d'une méthode intergouvernementale n'est rien d'autre que la traduction d'une situation ancienne. Depuis longtemps, c'est comme cela que fonctionne l'UE dans les domaines les plus sensibles, alors que l'interdépendance entre les pays membres est d'ores et déjà très grande.

Si on ajoute à ces éléments le manque de clarté du fonctionnement de l'UE pour les acteurs extérieurs, il n'est pas étonnant que les marchés restent méfiants et continuent de sanctionner les décisions européennes. Le fait que la Banque centrale ne puisse pas réellement intervenir en dehors des marchés secondaires pour soutenir les pays membres et que le FMI doive intervenir dans les dispositifs de soutien mis en place est source de perplexité pour de nombreux observateurs, qui y voient d'inutiles complications et un facteur de fragilité supplémentaire.

\section{Les citoyens européens : peu européens et pas fédéralistes?}

38 La fragilité de l'Union européenne réside en partie dans le fait qu'elle n'est pas encore appropriée par les Européens eux-mêmes. Tant que cette situation durera, tant qu'une opinion publique européenne n'aura pas émergé, c'est l'intergouvernementalisme avec ses divisions et ses lenteurs qui prévaudra. Deux faits s'imposent aux observateurs : pour les citoyens européens, c'est la référence nationale qui continue de primer largement sur la référence européenne, sur un plan politique et identitaire; les sentiments des Européens vis-à-vis de l'UE et de ses institutions sont très mitigés. D'ailleurs, la montée en puissance des partis populistes dans divers pays membres de l'UE - partis qui n'hésitent pas à demander à sortir de l'euro - lors des plus récentes élections législatives est un signe fort, surtout en Autriche, au Danemark et aux Pays-Bas et dans une moindre mesure en Allemagne, Belgique, Grèce, Hongrie, Italie, Portugal, République tchèque et Slovénie.

L'existence à la fois de traditions politiques et partisanes très prégnantes et de divergences idéologiques fortes entre les nations européennes est évidemment un obstacle difficile à surmonter dans la construction européenne. Les opinions publiques restent profondément nationales, ce qui rend difficile des avancées politiques majeures, d'autant plus que les responsables politiques ne sont comptables de leur action que vis-àvis de leur propre électorat national. Les peuples européens ont des représentations divergentes de l'Europe. C'est ce que montrent plusieurs enquêtes d'opinion réalisées 
depuis les années 1990. L'analyse de leurs résultats permet de distinguer deux grands types de situations :

- dans certains pays, généralement ceux de l'Europe centrale et de l'Europe occidentale, la population se reconnaît majoritairement comme européenne. Cela signifie que l'Europe est devenue un référent identitaire, même si elle ne vient le plus souvent qu'en deuxième position derrière la référence nationale ;

- dans d'autres pays, principalement ceux de l'Europe orientale autrefois soviétique, l'image de l'Europe est généralement bonne. Mais, très souvent, elle n'est pas reconnue comme une référence identitaire, même secondaire.

D'après une enquête réalisée par l'Union européenne en mai 2010, l'identité nationale vient toujours en tête dans la définition que les citoyens des pays membres de l'UE donnent d'eux-mêmes (illustration 6). Ce qui n'empêche pas $62 \%$ des personnes interrogées de se sentir citoyennes de l'Union ( $21 \%$ se disaient « tout à fait » européennes et $41 \%$ «plutôt» européennes). Et malgré l'émergence - lente - de l'Europe comme référence identitaire commune, diverses autres enquêtes ont montré que les Européens de l'ouest et du centre ont une vision finalement très nationale de l'Europe. Selon un sondage réalisé en 2003 par l'institut français de sondage CSA (en Allemagne, en Espagne, en France, en Grande-Bretagne, en Italie et en Pologne), il serait totalement injustifié de parler d'une mémoire européenne commune. Les personnes interrogées ont très souvent montré que leur représentation de l'Europe et de son histoire se construisait à partir de références nationales. Cela signifie qu'il n'existe pas encore un lien fort et direct entre l'Europe et les individus. La nation continue de s'intercaler entre les deux.

Illustration 6 - Nationalisme et européanité dans les pays européens

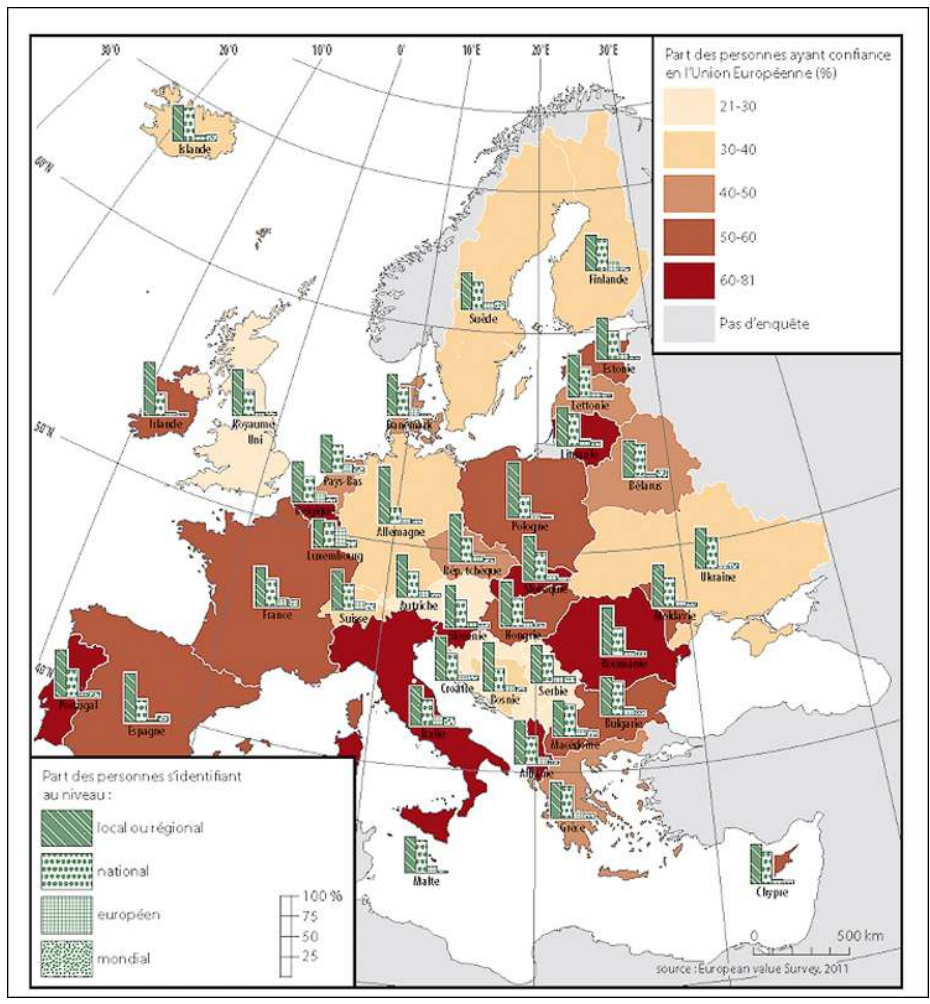

Source : European value Survey, 2011 ; conception : Yann Richard, réalisation : Florence Bonnaud. 
41 Notons enfin l'écart qui existe entre la géographie de la confiance dans l'Union européenne et celle de l'identité européenne. La confiance dans les institutions européennes montre une géographie très contrastée : elle est élevée dans la plupart des pays de l'Est de l'Union européenne et dans d'autres nations périphériques ayant largement bénéficié de leur intégration à l'Europe (Espagne, Portugal, Grèce ou Irlande) ainsi qu'en France, et dans les pays du Benelux ; elle est faible dans les pays nordiques, en Allemagne, en Autriche et surtout au Royaume-Uni où elle n'atteint que $20 \%$ hors Irlande du Nord. La géographie de l'identification à l'Europe est très différente, en partie l'inverse de la confiance dans les institutions européennes : elle atteint ses plus hauts niveaux dans l'Europe du Nord-Ouest jusque dans l'Italie du Nord et est plutôt faible dans une grande partie de la périphérie. Tout se passe comme si les nations prospères montraient une forte européanité sans pour autant souhaiter le renforcement de l'Europe politique. Toutefois, cela ne représente qu'une partie de la réalité : l'Italie du Nord, la France et le Benelux, nations prospères, ont à la fois des niveaux élevés d'identification à l'Europe et une confiance élevée dans les institutions européennes. Quoi qu'il en soit, la combinaison de fortes identifications nationales, de la faiblesse de l'identité européenne et d'une confiance limitée dans l'Europe politique illustre les difficultés de la construction européenne. C'est d'autant plus vrai qu'il existe de grands écarts sur ces questions entre les nations européennes, si bien qu'il semble difficile de s'accorder sur des objectifs communs.

Les enquêtes d'opinion récentes de l'Eurobaromètre confirment les enquêtes susmentionnées :

- en septembre 2011, l'Union européenne n'est vue comme l'acteur le plus à même de résoudre les problèmes économiques que par $22 \%$ des personnes interrogées, pas très loin devant les gouvernements nationaux (20\%);

- l'image de l'UE est d'ailleurs de plus en plus négative dans l'opinion : elle recueillait $52 \%$ d'avis positifs en septembre 2007, mais $31 \%$ en août 2011. Et les avis négatifs sont passés de $15 \%$ à $26 \%$. 
Illustration 7 - Avez-vous confiance dans ces institutions?

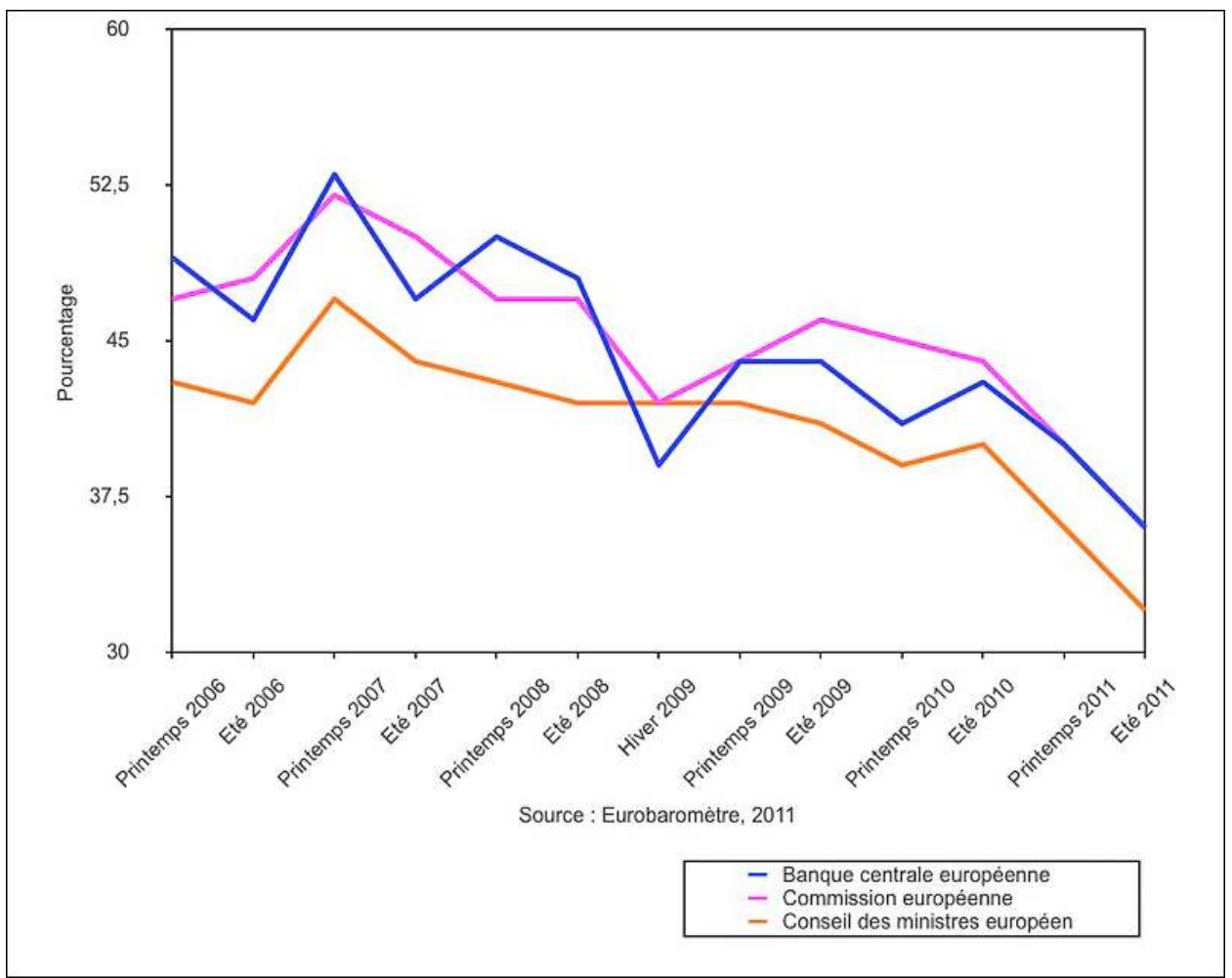

Source : Eurobaromètre, 2011. 


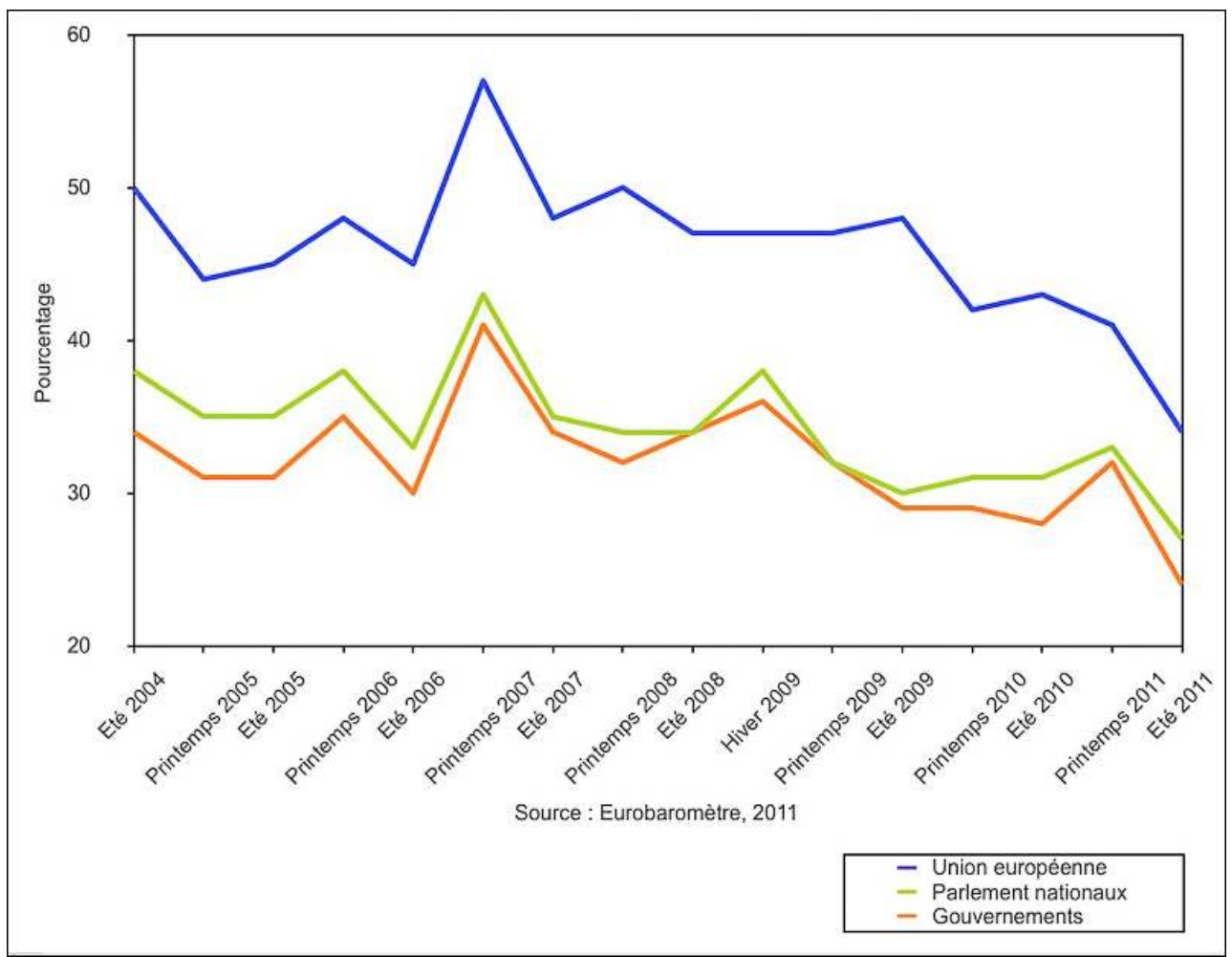

Source : Eurobaromètre, 2011

- la confiance dans toutes les institutions européennes a baissé de façon spectaculaire depuis 2007. Plus grave sans doute, le niveau de confiance varie considérablement d'un pays à l'autre. En septembre 2011, le niveau de confiance vis-à-vis du Conseil des ministres européens varie entre $13 \%$ (Royaume-Uni) et $49 \%$ en Roumanie. Pour la Commission européenne, ces chiffres variaient entre $14 \%$ (Royaume-Uni toujours) et $56 \%$ au Luxembourg;

- l'Union européenne est perçue comme non démocratique dans beaucoup de pays membres : Grèce, Italie, Autriche, Portugal, Slovaquie, Royaume-Uni. Plus de $50 \%$ des personnes interrogées le pensent en Bulgarie, au Danemark, à Chypre, en Lettonie, au Luxembourg, en Pologne, en Roumanie et en Suède. Enfin, l'opinion publique des pays du Sud estime en majorité que l'UE ne prend pas leur intérêt assez en compte ;

- le soutien à l'UEM et à l'Euro est variable aussi : les moins favorables sont les Britanniques (15\% d'opinions favorables), les Tchèques (22\%), les Suédois (23\%) et les Danois (29\%). Mais, plus préoccupant pour les décideurs politiques favorables à l'intégration économique, aucune opinion publique ne soutient une fiscalité européenne. Sans surprise, ce sont les opinions des pays membres du sud et de certains pays de l'est qui y sont les moins défavorables : Italie, Espagne, Lituanie, Portugal, Slovaquie. 
Illustration 9 - Etes-vous favorable à une union économique et monétaire ?

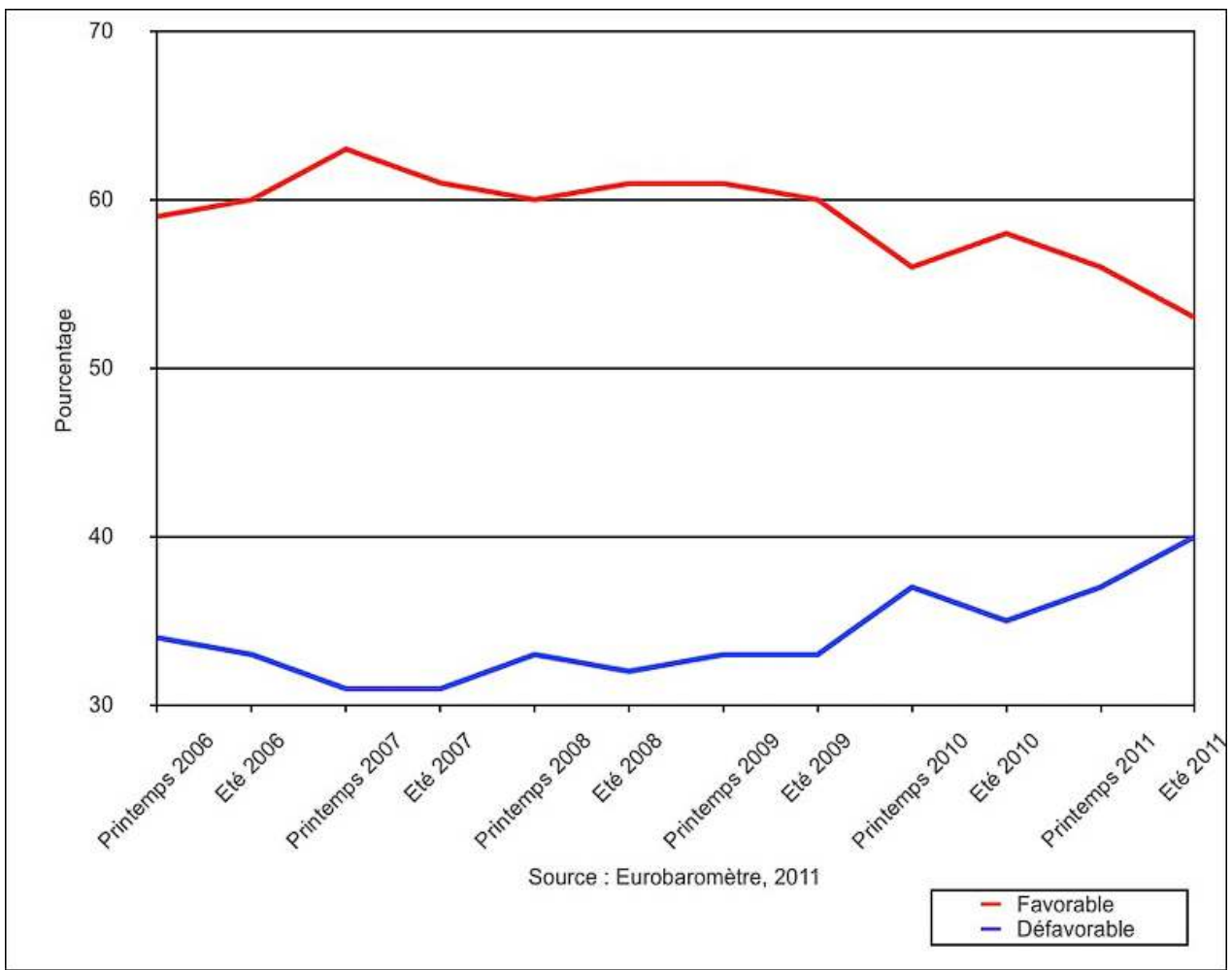

Source : Eurobaromètre, 2011.

- en revanche, il y a plus de soutien pour une politique européenne de l'emploi dans quelques pays mais avec des fortes inégalités, de même pour la sécurité sociale, pour la réduction de la dette publique, etc.

- ce qui n'empêche pas une majorité des citoyens européens d'être très favorables à une plus grande coordination des politiques, à plus de $50 \%$ dans 18 pays. Si on additionne les personnes très favorables et les personnes plutôt favorables, la moyenne de l'UE monte à $89 \%$ et les pourcentages les plus bas sont très élevés (76\% en Autriche et $82 \%$ au RoyaumeUni). L'opinion n'est pas fédéraliste mais semble favorable à un renforcement de la gouvernance intergouvernementale.

\section{Conclusion}

La crise ne doit pas faire oublier que l'UE pèse lourd dans la mondialisation (Didelon, Grasland, Richard, 2009). Elle représente environ $20 \%$ du PIB mondial. Son revenu national brut est supérieur à celui des États-Unis. Elle est la première puissance commerciale mondiale, la première origine et la première source des investissements internationaux, même en excluant les flux internes. Elle demeure très attractive puisqu'elle est la première destination mondiale des migrations internationales et des étudiants internationaux. Enfin et surtout, son image est excellente dans les nombreuses opinions publiques de nombreux pays. Mais elle est très fragile car ses divisions politiques et ses disparités économiques font d'elle une puissance en puissance dans de nombreux domaines. 
44 au bout d'une certaine logique fondée sur les idées des pères fondateurs : faire avancer l'intégration par la création de solidarités de fait, par l'instauration de règles communes et par le partage de la souveraineté dans un nombre croissant de domaines. Cette logique a été longtemps fondée sur des avancées techniques et n'a pas été suffisamment construite sur l'implication des citoyens européens eux-mêmes. Peut-elle continuer à fonctionner comme cela? Tous les observateurs, qu'ils soient favorables à l'intégration européenne ou non, sont d'accord sur ce point: il n'est pas possible d'avancer en conservant un modèle d'intégration déséquilibré. En effet, s'intégrer sur le plan monétaire rend les économies tellement interdépendantes que cela suppose qu'elles convergent sur le plan politique. Comment peut-on continuer à utiliser la même monnaie lorsque les performances macroéconomiques sont si inégales et les politiques économiques si différentes et peu complémentaires? Au nom de la cohérence et de l'efficacité, si l'on souhaite créer des solidarités de fait et des interdépendances monétaires et économiques entre les pays membres d'un espace commun, certaines politiques doivent être plus coordonnées voire à terme communautarisées. Ou bien l'Union et la zone euro doivent éclater pour sortir les pays membres de leur paralysie. Mais rien ne dit qu'ils en sortiraient plus forts. Ce n'est pas une affirmation idéologique, mais une simple question de logique et de cohérence. C'est l'un ou l'autre. L'entre deux n'est pas tenable.

La fragilité excessive de l'UE et de la zone euro vient de leurs disparités et de leurs divisions internes. Elle montre aussi que la fragilité structurelle de l'Union se retrouve à presque tous les étages. Et on peut se demander si le nouveau traité européen, tant souhaité par Mme Merkel, apportera des améliorations. Comme d'autres mesures de traitement de la crise, il met en avant davantage le principe de solidité financière que la croissance et l'emploi et suscite de nombreuses oppositions, soulignant la fragilité du leadership allemand ou franco-allemand. Il y a pourtant urgence à trouver un nouveau modèle européen plus efficace et plus lisible pour les citoyens européens eux-mêmes et pour les autres acteurs du jeu international. Ces derniers sont parfois découragés par la complexité dissuasive et la lenteur de certaines procédures européennes (Lynch, 2006), ou perçoivent davantage ses fragilités que ses atouts (Lisbonne-de Vergeron, 2012). Une intégration approfondie apporterait sans doute un surcroît d'efficacité. C'est aussi une condition préalable pour continuer de peser dans les négociations internationales face à des pays émergents qui revendiquent un rôle accru dans un système mondial polycentrique ou la puissance est plus diffuse qu'autrefois (Foucher, 2008). La mondialisation est, entre autres choses, une affaire de rapport de force, une sorte de « bataille » de normes et de règlementations (Laïdi, 2005). L'échec cuisant de l'UE lors des négociations sur le climat à Copenhague devrait être un signal d'alarme car il a confirmé que les Européens ne pèsent pas aussi lourds qu'ils le pensent dans certains domaines. Les Européens doivent se demander s'ils ont plus de chance de bien figurer dans cette «bataille » en restant divisés ou en poursuivant leur intégration. La crise est là pour le rappeler. 


\section{BIBLIOGRAPHIE}

Battistella D., Smouts M.-C., Vennesson, P., 2006. Dictionnaire des relations internationales. Paris, Dalloz, $2^{\mathrm{e}}$ édition.

Bertoncini Y., Chopin T., Dulphy A., Kahn S., Manigand C. (dir.), 2008. Dictionnaire critique de l'Union européenne. Paris, Armand Colin.

Boyer R., 2004. Une théorie du capitalisme est-elle possible ? Paris, Odile Jacob.

Braudel F., 1979. Civilisation matérielle, économie et capitalisme. 3 vol., Paris, Armand Colin.

Carroué L., 2008. Crise des subprimes : fin de l'hégémonie américaine. Images économiques du monde 2009 , p. 2-18.

Carroué L., 2009a. La crise économique et financière états-unienne : enjeux géographiques et géopolitiques. Hérodote, $\mathrm{n}^{\circ}$ 132, p. 104-127.

Carroué L., 2009b. Crise mondiale, crise sociale : les territoires dans la tourmente. Images économiques du monde 2010, p. 2-23.

Chambon J.-L., David J.H, 2009. Repenser la planète finance. Paris, Les Échos/Eyrolles.

Chopin T., Foucher, M. (dir.), 2011. Rapport Schuman sur l'Europe. L'état de l'Union 2011. Fondation Robert Schuman.

Colin S., 2010. La Chine en temps de crise. Images économiques du monde 2011, p. 8-27.

Dervi K., 2009. La crise économique mondiale : enjeux et réformes. Politique étrangère, p. 11-22.

Didelon C., Grasland Cl. (dir.), 2006. Europe in the World. ESPON Program, project 3.4.1, Rapport destiné à la Commission des Communautés européennes, DG Regio, www.espon.eu/

Didelon C., Grasland C., Richard Y. (dir.), 2009. Atlas de l'Europe dans le Monde. Paris La documentation française, Montpellier, Reclus.

Didelon C., Richard Y., Van Hamme G., 2011. Le territoire européen, Paris, PUF.

Foucher M., 2008. Retour des frontières, pérennité des États dans le monde polycentrique. In Gentelle P, Géopolitique du monde contemporain, Paris, Nathan, p. 269-281.

Giraud P.N., 2009. 1929-1990-2008 : les leçons des crises passées face à la crise actuelle. Politique étrangère, $\mathrm{n}^{\circ} 2$, p. 367-376.

Images économiques du monde. Géopolitique, géoéconomie 2012. Paris, Armand Colin, 2011.

Kébabdjian G., 1999. Les théories de l'économie politique internationale. Paris, Seuil.

Laïdi Z., 2005. La norme sans la force. L'énigme de la puissance européenne. Paris, Les Presses de Sciences Po.

Lisbonne-de Vergeron, K., 2012. L'Europe vue de Chine et d'Inde depuis la crise : nouvelles perspectives des grands émergents asiatiques. Paris, Fondation Robert Schuman.

Lynch D., 2006. Russian Perceptions of the CFSP/ESDP. Analysis Paper, Institute for security Studies, http://www.iss.europa.eu/uploads/media/analy145.pdf 
OFCE, 2011. Zone euro : coupée en deux. Perspectives 2011-2012 pour l'économie européenne. Revue de l'OFCE, 2011, n 117, p. 43-89.

Sapir J., 2006. La Crise de l'Euro : erreurs et impasses de l'Européisme. Perspectives Républicaines, $\mathrm{n}$ -2, 2006, p. 69-84.

Sapir J., 2008. La Crise financière et ses enjeux. URL: http://contreinfo.info/article.php3? id_article=2242.

Van Hamme G., 2011a. Les inégalités de développement territorial. In Didelon C., Richard Y., Van Hamme G., Le territoire européen, Paris, PUF, p. 67-94.

Van Hamme G., 2011b. Le poids persistant des nations ou les résistances à l'intégration. In Didelon C., Richard Y., Van Hamme G., Le territoire européen, Paris, PUF, p. 133-154.

\section{NOTES}

1. Les baisses de PIB en glissement annuel au deuxième trimestre 2009 ont été même plus fortes :

- 16,6 \% en l'Estonie, - 19,6 \% en Lettonie et - 20,2 \% en Lituanie.

2. «Vingt ans après, les Européens refondent Maastricht afin de restaurer la confiance » Les Echos, 12/12/2011. http://www.lesechos.fr/economie-politique/monde/ dossier/0201699066506/0201790520822-vingt-ans-apres-les-europeens-refondent-maastrichtafin-de-restaurer-la-confiance-261710.php

\section{RÉSUMÉS}

L'Union européenne est durement affectée par la crise des dettes souveraines. Cette crise peut être interprétée comme la conséquence de plusieurs faits importants : une absence de leadership européen, des inégalités fortes parfois anciennes entre les performances macroéconomiques des pays membres, des faiblesses structurelles déjà observables avant la crise dans certains pays. Mais le principal facteur de fragilité de l'Union européenne réside dans sa division interne. Tant que la problématique de l'intégration européenne ne sera pas appropriée par les citoyens européens eux-mêmes et tant que certaines politiques ne seront pas davantage coordonnées, l'Union restera sur le fil du rasoir. Cette crise met en lumière l'essoufflement d'un certain modèle d'intégration. Dans cet article, on explore ces problématiques avec un regard géographique.

\section{INDEX}

Mots-clés : crise économique, division, État, fédéralisme, gouvernance, intégration, nation, Union européenne, zone euro 


\section{AUTEUR}

\section{YANN RICHARD}

Yann Richard est Professeur de géographie à l'Université Paris I et membre de l'UMR Prodig.

Yann.Richard@univ-paris1.fr 